\title{
THE ROLE OF HERMITE POLYNOMIALS IN ASYMPTOTIC ANALYSIS
}

\author{
NICO M. TEMME \\ CWI, P.O. Box 94079, 1090 GB Amsterdam, The Netherlands \\ e-mail: nicotQcwi.nl \\ JOSÉ L. LÓPEZ \\ Dpto. Matematica e Informatica, Universidad Publica de Navarra, \\ Campus de Arrosadia s/n, 31006-Pamplona, Spain \\ e-mail: jl.lopezQunavarra.es
}

\begin{abstract}
Hermite polynomials are considered as approximants in asymptotic representations of certain other polynomials. Examples are given for polynomials from the Askey scheme of hypergeometric orthogonal polynomials. We also mention that Hermite polynomials can be used as main approximants in uniform asymptotic representations of certain types of integrals and differential equations.
\end{abstract}

\section{Introduction}

Hermite polynomials show up in several problems of asymptotic analysis. We consider three different instances where these classical orthogonal polynomials can be used as main approximants:

1. As limits of other polynomials such as Laguerre and Jacobi orthogonal polynomials, but also of generalized Bernoulli polynomials. We explain that these limits may be derived from asymptotic representations in which Hermite polynomials occur.

2. In turning point problems for second order linear differential equations. In particular the Hermite polynomials can be used when two nearby turning points are present.

3. For functions defined as an integral in which the saddle points follow a certain pattern for certain values of the parameters

In all three cases, asymptotic representations of polynomials are considered in terms of Hermite polynomials. In the second and third case the Hermite polynomials arise as special cases of another set of special functions, the Weber parabolic cylinder functions, which can be used in similar and more general problems of asymptotic analysis. 
We concentrate on the first topic; in particular we give examples from the Askey scheme of hypergeometric polynomials. We give examples in which Hermite polynomials are used in asymptotic approximations, and we also give approximations in terms of other polynomials.

\section{Limits between orthogonal polynomials}

It is well known that the Hermite polynomials play a crucial role in certain limits of the classical orthogonal polynomials. For example, the ultraspherical (Gegenbauer) polynomials $C_{n}^{\gamma}(x)$, which are defined by the generating function

$$
\left(1-2 x w+w^{2}\right)^{-\gamma}=\sum_{n=0}^{\infty} C_{n}^{\gamma}(x) w^{n}, \quad-1 \leq x \leq 1, \quad|w|<1,
$$

have the well-known limits

$$
\lim _{\gamma \rightarrow \infty} \frac{C_{n}^{\gamma}(x)}{C_{n}^{\gamma}(1)}=x^{n}, \quad \lim _{\gamma \rightarrow \infty} \gamma^{-n / 2} C_{n}^{\gamma}(x / \sqrt{\gamma})=\frac{1}{n !} H_{n}(x) .
$$

The first limit shows that the zeros of $C_{n}^{\gamma}(x)$ tend to the origin if the order $\gamma$ tends to infinity. The second limit is more interesting; it gives the relation with the Hermite polynomials if the order becomes large and the argument $x$ is properly scaled.

For the Laguerre polynomials, which are defined by the generating function

$$
(1-w)^{-\alpha-1} e^{-w x /(1-w)}=\sum_{n=0}^{\infty} L_{n}^{\alpha}(x) w^{n}, \quad|w|<1,
$$

$\alpha, x \in \mathbb{C}$, similar results are

$$
\begin{aligned}
& \lim _{\alpha \rightarrow \infty} \alpha^{-n} L_{n}^{\alpha}(\alpha x)=\frac{(1-x)^{n}}{n !}, \\
& \lim _{\alpha \rightarrow \infty} \alpha^{-n / 2} L_{n}^{\alpha}(x \sqrt{\alpha}+\alpha)=\frac{(-1)^{n} 2^{-n / 2}}{n !} H_{n}(x / \sqrt{2}) .
\end{aligned}
$$

This again gives insight in the location of the zeros for large values of the order $\alpha$, and the relation with the Hermite polynomials if the order becomes large and $x$ is properly scaled.

Many methods are available to prove these and other limits. In this paper we concentrate on asymptotic relations between the polynomials, from which the limits follow as special cases. 


\section{The Askey scheme}

In Koekoek \& Swarttouw (1998) many relations are given for hypergeometric orthogonal polynomials and their $q$-analogues, including limit relations between many polynomials. In Figure 1 we show examples for which limit relations between neighboring polynomials are available, but many other limit relations are mentioned in Koekoek \& Swarttouw (1998), Godoy et al. (1998) and Ronveaux et al. (1998)

\section{Askey Scheme of Hypergeometric \\ Orthogonal Polynomials}

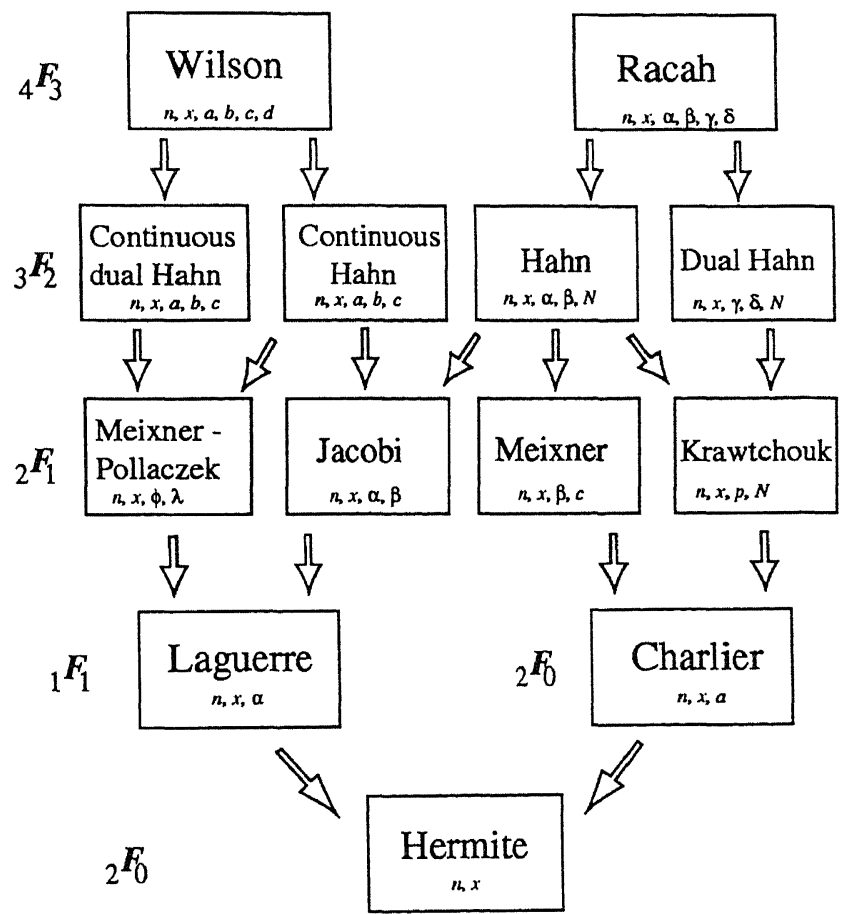

Figure 1. The Askey scheme for hypergeometric orthogonal polynomials, with indicated limit relations between the polynomials.

In López and Temme (1999a, 1999b) we have given several asymptotic relations between polynomials and Hermite polynomials. In the first paper we 
considered Gegenbauer polynomials, Laguerre polynomials, Jacobi polynomials and Tricomi-Carlitz polynomials. In the second paper we have considered generalized Bernoulli polynomials, generalized Euler polynomials, generalized Bessel polynomials and Buchholz polynomials.

The method for all these cases is the same and we observe that the method also works for polynomials outside the class of hypergeometric polynomials. The method is different from the one described in Godoy et al. (1998), where also more terms in the limit relation are constructed in order to obtain more insight in the limiting process.

In current research we investigate if other limits in the Askey scheme can be replaced by asymptotic results. Until now we verified all limits from the third level to the fourth (Laguerre and Charlier) and the fifth level (Hermite). Several limits are new, and all results have full asymptotic expansions.

\section{Asymptotic representations}

Starting point is a generating series

$$
F(x, w)=\sum_{n=0}^{\infty} p_{n}(x) w^{n},
$$

$F$ is a given function, which is analytic with respect to $w$ at $w=0$, and $p_{n}$ is independent of $w$.

The relation (4.1) gives for $p_{n}$ the Cauchy-type integral

$$
p_{n}(x)=\frac{1}{2 \pi i} \int_{\mathcal{C}} F(x, w) \frac{d w}{w^{n+1}}
$$

where $\mathcal{C}$ is a circle around the origin inside the domain where $F$ is analytic (as a function of $w$ ).

We write

$$
F(x, w)=e^{A w-B w^{2}} f(x, w),
$$

where $A$ and $B$ do not depend on $w$. This gives

$$
p_{n}(x)=\frac{1}{2 \pi i} \int_{\mathcal{C}} e^{A w-B w^{2}} f(x, w) \frac{d w}{w^{n+1}} .
$$

Because $f$ is also analytic (as a function of $w$ ), we can expand

$$
f(x, w)=e^{-A w+B w^{2}} F(x, w)=\sum_{k=0}^{\infty} c_{k} w^{k},
$$


that is,

$$
f(x, w)=1+\left[p_{1}(x)-A\right] w+\left[p_{2}(x)-A p_{1}(x)+B+\frac{1}{2} A^{2}\right] w^{2}+\ldots
$$

if we assume that $p_{0}(x)=1$ (which implies $c_{0}=1$ ).

We substitute (4.3) in (4.2). The Hermite polynomials have the generating function

$$
e^{2 x w-w^{2}}=\sum_{n=0}^{\infty} \frac{H_{n}(x)}{n !} w^{n}, \quad x, w \in \mathbb{C},
$$

which gives the Cauchy-type integral

$$
H_{n}(x)=\frac{n !}{2 \pi i} \int_{\mathcal{C}} e^{2 x z-z^{2}} z^{-n-1} d z
$$

where $\mathcal{C}$ is a circle around the origin and the integration is in positive direction. The result is the finite expansion

$$
p_{n}(x)=z^{n} \sum_{k=0}^{n} \frac{c_{k}}{z^{k}} \frac{H_{n-k}(\xi)}{(n-k) !}, \quad z=\sqrt{B}, \quad \xi=\frac{A}{2 \sqrt{B}},
$$

because terms with $k>n$ do not contribute in the integral in (4.2).

In order to obtain an asymptotic property of (4.5) we take $A$ and $B$ such that $c_{1}=c_{2}=0$. This happens if we take

$$
A=p_{1}(x), \quad B=\frac{1}{2} p_{1}^{2}(x)-p_{2}(x) .
$$

As we will show, the asymptotic property follows from the behavior of the coefficients $c_{k}$ if we take a parameter of the polynomial $p_{k}(x)$ large. We use the following lemma, and explain what happens by considering a few examples. Lemma 4.1 Let $\phi(w)$ be analytic at $w=0$, with Maclaurin expansion of the form

$$
\phi(w)=\mu w^{n}\left(a_{0}+a_{1} w+a_{2} w^{2}+\ldots\right),
$$

where $n$ is a positive integer and $a_{k}$ are complex numbers that do not depend on the complex number $\mu, a_{0} \neq 0$. Let $c_{k}$ denote the coefficients of the power series of $f(w)=e^{\phi(w)}$, that is,

$$
f(w)=e^{\phi(w)}=\sum_{k=0}^{\infty} c_{k} w^{k} .
$$

Then $c_{0}=1, c_{k}=0, k=1,2 \ldots, n-1$ and

$$
c_{k}=\mathcal{O}\left(|\mu|^{\lfloor k / n\rfloor}\right), \quad \mu \rightarrow \infty \text {. }
$$


Proof The proof follows from expanding

$$
\begin{aligned}
\sum_{k=0}^{\infty} c_{k} w^{k} & =e^{\phi(w)}=\sum_{k=0}^{\infty} \frac{[\phi(w)]^{k}}{k !} \\
& =\sum_{k=0}^{\infty} \frac{\mu^{k} w^{k n}}{k !}\left(a_{0}+a_{1} w+a_{2} w^{2}+\ldots\right)^{k},
\end{aligned}
$$

and comparing equal powers of $w$.

\subsection{Ultraspherical polynomials}

The generating function is

$$
F(x, w)=\left(1-2 x w+w^{2}\right)^{-\gamma}=\sum_{n=0}^{\infty} C_{n}^{\gamma}(x) w^{n}
$$

with

$$
C_{0}^{\gamma}(x)=1, \quad C_{1}^{\gamma}(x)=2 \gamma x, \quad C_{2}^{\gamma}(x)=2 \gamma(\gamma+1) x^{2}-\gamma
$$

Hence,

$$
A=C_{1}^{\gamma}(x)=2 x \gamma, \quad B=\frac{1}{2}\left[C_{1}^{\gamma}(x)\right]^{2}-C_{2}^{\gamma}(x)=\gamma\left(1-2 x^{2}\right),
$$

and we can write

$$
C_{n}^{\gamma}(x)=z^{n} \sum_{k=0}^{n} \frac{c_{k}}{z^{k}} \frac{H_{n-k}(\xi)}{(n-k) !}
$$

where

$$
z=\sqrt{\gamma\left(1-2 x^{2}\right)}, \quad \xi=\frac{x \gamma}{z}
$$

We have

$$
c_{0}=1, \quad c_{1}=c_{2}=0, \quad c_{3}=\frac{2}{3} \gamma x\left(4 x^{2}-3\right) .
$$

Higher coefficients follow from a recursion relation.

The function $f(x, w)$ of (4.3) has the form $f(x, w)=e^{\phi(x, w)}$, where $\phi(x, w)=\gamma w^{3}\left(a_{0}+a_{1} w+a_{2} w^{2}+\ldots\right)$. By using Lemma 4.1 and $\xi=\mathcal{O}(\sqrt{\gamma})$ we conclude that the sequence $\left\{\phi_{k}\right\}$ with $\phi_{k}=c_{k} / z^{k} H_{n-k}(\xi)$ has the following asymptotic structure:

$$
\phi_{k}=\mathcal{O}\left(\gamma^{n / 2+\lfloor k / 3\rfloor-k}\right), \quad k=0,1,2, \ldots
$$


This explains the asymptotic nature of the representation in (4.6) for large values of $\gamma$, with $x$ and $n$ fixed.

To verify the limits given in Section 2, we first write $x$ in terms of $\xi$ :

$$
x=\frac{\xi}{\sqrt{\gamma+2 \xi^{2}}} \text {. }
$$

With this value of $x$ we can verify that

$$
c_{k} / z^{k}=o(1), \quad \gamma \rightarrow \infty, \quad k>0,
$$

and in fact we have the limit

$$
\lim _{\gamma \rightarrow \infty} \frac{\gamma^{n}}{\left(\gamma+2 x^{2}\right)^{n / 2}} C_{n}^{\gamma}\left(\frac{x}{\sqrt{\gamma+2 x^{2}}}\right)=\frac{1}{n !} H_{n}(x) .
$$

\subsection{Laguerre polynomials}

We take as generating function (see (2.1))

$$
F(x, w)=(1+w)^{-\alpha-1} e^{w x /(1+w)}=\sum_{n=0}^{\infty}(-1)^{n} L_{n}^{\alpha}(x) w^{n}
$$

We have

$$
\begin{aligned}
& L_{0}^{\alpha}(x)=1, \quad L_{1}^{\alpha}(x)=\alpha+1-x \\
& L_{2}^{\alpha}(x)=\frac{1}{2}\left[(\alpha+1)(\alpha+2)-2(\alpha+2) x+x^{2}\right]
\end{aligned}
$$

which gives

$$
A=x-\alpha-1, \quad B=x-\frac{1}{2}(\alpha+1)
$$

and we obtain

$$
L_{n}^{\alpha}(x)=(-1)^{n} z^{n} \sum_{k=0}^{n} \frac{c_{k}}{z^{k}} \frac{H_{n-k}(\xi)}{(n-k) !}
$$

where

$$
z=\sqrt{x-\frac{1}{2}(\alpha+1)}, \quad \xi=\frac{x-\alpha-1}{2 z}
$$

We have

$$
c_{0}=1, \quad c_{1}=c_{2}=0, \quad c_{3}=\frac{1}{3}(3 x-\alpha-1) .
$$

Higher coefficients follow from a recursion relation. The representation in (4.7) has an asymptotic character for large values of $|\alpha|+|x|$. It is not difficult to verify that the limits given in (2.2) follow from (4.7). 


\section{Meixner-Pollaczek polynomials into Laguerre polynomials}

We give an example on how to use Laguerre polynomials for approximating other polynomials.

Lemma 5.1 Let the polynomials $p_{n}(x)$ be defined by the generating function

$$
F(x, w)=\sum_{n=0}^{\infty} p_{n}(x) w^{n}
$$

where $F(x, w)$ is analytic in $w=0$ and $F(x, 0)=1$. Let the coefficients $c_{k}(x)$ be defined by the expansion

$$
e^{-A w /(B w-1)}(1-B w)^{C+1} F(x, w)=\sum_{k=0}^{\infty} c_{k}(x) w^{k}, \quad c_{0}=1,
$$

where $A, B$ and $C$ do not depend on $w$. Then $p_{n}(x)$ can be represented as the finite sum

$$
p_{n}(x)=B^{n / 2} \sum_{k=0}^{n} \frac{c_{k}(x)}{B^{k / 2}} L_{n-k}^{(C)}(\xi), \quad \xi=\frac{A}{B},
$$

where $L_{n}^{\alpha}(x)$ are the Laguerre polynomials. Moreover, $A, B$ and $C$ can be chosen such that $c_{1}=0, c_{2}=0, c_{3}=0$.

Proof Use the Cauchy integral of $p_{n}(x)$ and of the Laguerre polynomials.

For the Meixner-Pollaczek polynomials we have the generating function:

$$
F(w)=\left(1-e^{i \phi} w\right)^{-\lambda+i x}\left(1-e^{-i \phi} w\right)^{-\lambda-i x}=\sum_{n=0}^{\infty} P_{n}^{(\lambda)}(x ; \phi) w^{n} .
$$

From (2.1) it follows that

$$
G(w)=e^{A w /(B w-1)}(1-B w)^{-C-1}=\sum_{n=0}^{\infty} L_{n}^{(C)}(\xi) B^{n} w^{n},
$$

where $\xi=A / B$. We define $c_{k}$ by $f(w)=F(w) / G(w)=\sum_{k=0}^{\infty} c_{k} w^{k}$. Then the expansion for the Meixner-Pollaczek polynomials reads

$$
P_{n}^{(\lambda)}(x ; \phi)=\sum_{k=0}^{n} B^{n-k} c_{k} L_{n-k}^{(C)}(\xi), \quad \xi=A / B .
$$

We write $x+i \lambda=r e^{i \theta}, \theta \in[0, \pi], r \geq 0$, and consider $r \rightarrow \infty$; the asymptotic results hold uniformly with respect to $\theta$. 
First we consider a simple case by taking $B=1$ and $C=\alpha$, and solve $c_{1}=0$ for $A$. This gives

$$
A=\alpha+1-2 \lambda \cos \phi-2 x \sin \phi .
$$

The first coefficients $c_{k}$ are given by

$$
c_{0}=1, \quad c_{1}=0, \quad c_{2}=x \sin 2 \phi+\lambda \cos 2 \phi-2(x \sin \phi+\lambda \cos \phi)+\frac{1}{2} \alpha .
$$

The first term approximation can be written as

$$
P_{n}^{(\lambda)}(x ; \phi)=\left[L_{n}^{(\alpha)}(\xi)+\mathcal{O}\left(r^{n-1}\right)\right], \quad \xi=A .
$$

In this case a limit can be obtained by putting $\lambda=(\alpha+1) / 2$. Then we have $c_{k}=\mathcal{O}\left(\phi^{2}\right)$ as $\phi \rightarrow 0$ for $k \geq 2$, and we obtain

$$
\left.\lim _{\phi \rightarrow 0} P_{n}^{(\alpha+1) / 2}[(\alpha+1)(1-\cos \phi)-\xi) /(2 \sin \phi) ; \phi\right]=L_{n}^{(\alpha)}(\xi) .
$$

This includes the limit of the Askey scheme

$$
\lim _{\phi \rightarrow 0} P_{n}^{(\alpha+1) / 2}(-\xi /(2 \phi) ; \phi)=L_{n}^{(\alpha)}(\xi) .
$$

Next we solve $c_{1}=0, c_{2}=0$ for $A$ and $C$, with $B=1$. This gives

$$
\begin{aligned}
& A=2[x(\sin \phi-\sin 2 \phi)+\lambda(\cos \phi-\cos 2 \phi)], \\
& C=2[x(2 \sin \phi-\sin 2 \phi)+\lambda(2 \cos \phi-\cos 2 \phi)]-1 .
\end{aligned}
$$

and the first term approximation can be written as

$$
P_{n}^{(\lambda)}(x ; \phi)=\left[L_{n}^{(\alpha)}(\xi)+\mathcal{O}\left(r^{n-2}\right)\right], \quad \xi=A, \quad \alpha=C .
$$

as $r \rightarrow \infty$, uniformly with respect to $\theta$.

Solving $A=\xi, C=\alpha$ for $x$ and $\lambda$, we obtain

$$
\begin{aligned}
& \lambda=(1-\cos \phi) \xi+\frac{1}{2}(\alpha+1)(2 \cos \phi-1), \\
& x=\frac{2(\xi-\alpha-1) \cos ^{2} \phi+(\alpha+1-2 \xi) \cos \phi+\alpha+1-\xi}{2 \sin \phi} .
\end{aligned}
$$

Then $c_{3}=\frac{2}{3}(\alpha+1-2 \xi)(1-\cos \phi)$ and $c_{k}=\mathcal{O}\left(\phi^{2}\right)$ as $\phi \rightarrow 0$ for $k \geq 3$. We obtain the limit

$$
\lim _{\phi \rightarrow 0} P_{n}^{(\lambda)}(x ; \phi)=L_{n}^{(\alpha)}(\xi)
$$




\section{Methods based on differential equations}

The function

$$
U_{n}(x)=e^{-\frac{1}{2} x^{2}} H_{n}(x)
$$

satisfies the differential equation

$$
U^{\prime \prime}=p(x) U, \quad p(x)=x^{2}-(2 n+1) .
$$

The function $p(x)$ has two real zeros $\pm \sqrt{2 n+1}$, and all $n$ zeros of $H_{n}(x)$ are in the interval $(-\sqrt{2 n+1}, \sqrt{2 n+1})$.

Scaling parameters, we see that $V_{n}(t):=U_{n}(\sqrt{2 n+1} t)$ satisfies

$$
\frac{d^{2}}{d t^{2}} V=(2 n+1)^{2}\left(t^{2}-1\right) V
$$

The question now is, can we approximate the solutions of the equation

$$
\frac{d^{2}}{d t^{2}} W=\nu^{2}\left(t^{2}-\alpha^{2}\right) W+f(t) W, \quad \nu \rightarrow \infty,
$$

in terms of Hermite polynomials or related functions?

For more details on this method we refer to Olver (1980) and Temme (1990).

\section{Hermite-type approximations for integrals}

The Hermite polynomials can be represented in the form

$$
\frac{1}{n !} H_{n}(\xi \sqrt{2 n+1})=\frac{2^{n / 2}}{\left(n+\frac{1}{2}\right)^{n / 2} 2 \pi i} \int_{\mathcal{C}} e^{\left(n+\frac{1}{2}\right) \psi(t)} \frac{d t}{\sqrt{t}}
$$

where

$$
\psi(t)=2 \xi t-\ln t-\frac{1}{2} t^{2} .
$$

and the contour $\mathcal{C}$ runs from $t=-\infty$, ph $t=-\pi$, encircles the origin in positive direction, and terminates at $-\infty$, now with ph $t=+\pi$.

The saddle points of the integral are defined by the equation $\psi^{\prime}(t)=$ $2 \xi-1 / t-t=0$ and are given by

$$
t_{1,2}=\xi \pm \sqrt{\xi^{2}-1} .
$$

When $\xi= \pm 1$ the saddle points coalesce, and when $\xi \sim 1$ uniform Airy-type expansions can be derived. When $-1<\xi<1$ the saddle points are complex (on the unit circle); for these values of $\xi$, that is, if $-\sqrt{2 n+1}<x<\sqrt{2 n+1}$, 
zeros occur. When $\xi>1$ or $\xi<-1$ the saddle points are real, and the Hermite polynomials are non-oscillating. See Figure 2 for the location of the saddle points.

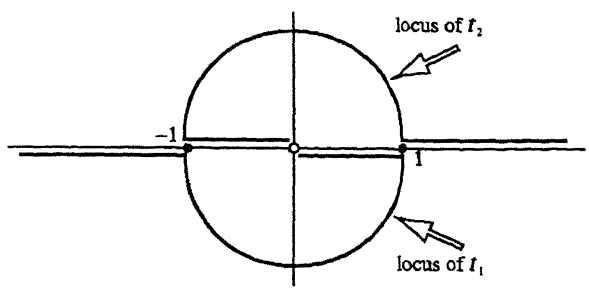

Figure 2. The location of the two saddle points $t_{1,2}$ defined in (7.2).

7.1 An expansion in terms of Hermite polynomials

We consider integrals of the form

$$
F_{\kappa}(\xi)=\frac{1}{2 \pi i} \int_{\mathcal{C}} e^{\kappa \Psi(t)} f(t) \frac{d t}{t}
$$

where

$$
\Psi(t)=2 \xi t-\rho^{2} \ln t-\frac{1}{2} t^{2} .
$$

We assume that $\kappa$ is a positive large parameter and that $\rho$ is positive. The contour $\mathcal{C}$ is as in (7.1).

The saddle points $t_{1,2}$ are now given by

$$
t_{1,2}=\xi \pm \sqrt{\xi^{2}-\rho^{2}} .
$$

For large values of $\kappa$ the function $F_{\kappa}(\xi)$ defined in (7.3) can be expanded in terms of parabolic cylinder functions. This asymptotic expansion holds uniformly with respect to $\xi \in \mathbb{R}$ and $\rho \in[0, \infty)$. For certain values of $\kappa$ and $\rho$ the parabolic cylinder functions reduce to Hermite polynomials.

For more details we refer to Temme (1986), Bo Rui \& Wong (1994), Jin \& Wong (1996), and Li \& Wong (1999).

\section{References}

1. Bo Rui \& R. Wong (1994). Uniform asymptotic expansion of Charlier polynomials, Methods Appl. Anal. 1, 294-313. 
2. E. Godoy, A. Ronveaux, A. Zarzo \& I. Area (1998). On the limit relations between classical continuous and discrete orthogonal polynomials, J. Comp. Appl. Math., 91, 97-105.

3. X.-S. Jin \& R. Wong (1997). Uniform asymptotic expansions for Meixner polynomials. Manuscript. Constructive Approximation 33, 119-127.

4. R. Koekoek \& R.F. Swarttouw (1998). The Askey-scheme of hypergeometric orthogonal polynomials and its $q$-analogue. Technical University Delft. Report 98-17.

5. X. Li \& R.Wong (1999). On the asymptotics of the Meixner-Pollaczek polynomials and their zeros. Submitted.

6. J.L. López \& N.M. Temme (1999a). Approximations of orthogonal polynomials in terms of Hermite polynomials. CWI Report MAS-R9901. Accepted for publication in Methods and Applications of Analysis.

7. J.L. López \& N.M. Temme (1999b). Hermite polynomials in asymptotic representations of generalized Bernoulli, Euler, Bessel and Buchholz polynomials. Submitted.

8. F.W.J. Olver (1980), Whittaker functions with both parameters large: Uniform approximations in terms of parabolic cylinder functions, Proc. Roy. Soc. Edinburgh, 86A, 213-234.

9. A. Ronveaux, A. Zarzo, I. Area \& E. Godoy (1998). Transverse limits in the Askey tableau, J. Comp. Appl. Math., 98, 327-335.

10. Temme, N.M. (1986). Laguerre polynomials: Asymptotics for large degree, CWI Report AM-R8610, Amsterdam.

11. N.M. Temme (1990). Polynomial asymptotic estimates of Gegenbauer, Laguerre, and Jacobi polynomials, 455-476 in Asymptotic and computational analysis, Proceedings, R. Wong (ed.), Lect. Notes Pure Appl. Math., 124, Marcel Dekker, New York.

12. N.M. Temme (1996), Special functions: An introduction to the classical functions of mathematical physics, Wiley, New York. ISBN 0-471-113131.

13. R. Wong (1989), Asymptotic approximations of integrals, Academic Press, New York. ISBN 0-12-762535-6. 